\title{
Line broadening in the PXRD patterns of layered hydroxides: The relative effects of crystallite size and structural disorder
}

\author{
GRACE S THOMAS and P VISHNU KAMATH* \\ Department of Chemistry, Central College, Bangalore University, Bangalore 560001 \\ e-mail: vishnukamath8@hotmail.com
}

\begin{abstract}
Layered hydroxides crystallize in a hexagonal structure and incorporate a number of different types of structural disorders as an exigency of anisotropic bonding. Structural disorder contributes to the non-uniform broadening of lines in the powder X-ray diffraction pattern. Common among the disorders are stacking faults, which broaden the $h 0 / / 0 k \ell$ reflections. Interstratification selectively broadens the $00 \ell$ reflections and turbostratic disorder broadens the $0 \mathrm{kl}$ reflections. The line broadening caused by structural disorder has to be discounted before estimates of particle size are made by applying the Scherrer formula.
\end{abstract}

Keywords. Layered double hydroxides; pyroaurite; structural disorder; stacking faults; turbostraticity; interstratification; polytypes.

\section{Introduction}

Powder X-ray diffraction (PXRD) is the most widely used technique to characterize a crystalline solid. The diffraction pattern comprises a number of sharp Bragg reflections corresponding to the different $d$ spacings of the solid. The broadening of peaks in the PXRD pattern beyond what is expected from instrumental factors is generally attributed to crystallite size effects. Small crystallites are responsible for the Scherrer broadening of lines. ${ }^{1}$ Any non-uniform broadening of lines is attributed to anisotropic crystallites. $^{2,3}$ The use of Scherrer broadening to estimate crystallite size has become very widespread in view of the contemporary interest in nanoparticulate materials.

However, this interpretation is fraught with many difficulties.

(1) When the material under investigation is disordered, structural disorder causes the broadening of Bragg reflections, which can be mistakenly attributed to crystallite size effects. This is especially true of layered materials, which incorporate a variety of structural disorders as an exigency of anisotropic bonding. Some of the commonly found disorders are stacking faults, turbostraticity and interstratification.

(2) Direct experimental measurement of crystallite size by electron microscopy is difficult when the

Dedicated to Prof J Gopalakrishnan on his 62nd birthday *For correspondence crystallites are not monodispersed or appear as aggregates. In such instances, crystallite size estimates obtained from PXRD remain experimentally unverified.

(3) When the material under investigation is a large band-gap insulator, there are no useful sizedependent properties that can be measured to verify the effect of crystallite size. In such instances, the likelihood of structural disorder contributing to line width in the PXRD pattern has to be discounted before Scherrer broadening can be applied to estimate crystallite size.

As part of our growing concern over these issues, we examined in earlier papers the effect of various kinds of disorder on the line width of Bragg reflections in the PXRD patterns of $\mathrm{Mg}(\mathrm{OH})_{2}{ }^{4}$ and $\mathrm{Ni}(\mathrm{OH})_{2}{ }^{5}$ We compared the effect of disorder with that of crystallite size to show that most preparations of divalent hydroxides are replete with structural disorder, and that the excessive and non-uniform broadening of lines in the PXRD patterns is on account of structural disorder rather than due to crystallite size effects.

We now extend these studies to another class of layered compounds, the layered double hydroxides (LDHs). ${ }^{6}$ The LDHs are obtained from $\mathrm{Mg}(\mathrm{OH})_{2}$ by the isomorphous substitution of a fraction $x$, of the $\mathrm{Mg}^{2+}$ ions by trivalent ions such as $\mathrm{Al}^{3+}$. This generates positively charged layers of the composition $\left[\mathrm{Mg}_{1-x} \mathrm{Al}_{x}(\mathrm{OH})_{2}\right]^{x+}$. These layers incorporate anions, $\mathrm{A}^{n-}\left(\mathrm{A}^{n-}=\mathrm{Cl}^{-}, \mathrm{CO}_{3}^{2-}, \mathrm{SO}_{4}^{2-}\right.$ and others $)$ and water molecules in between the layers for charge neutrality 
and stability. A number of divalent ions such as $\mathrm{Ca}$, $\mathrm{Co}, \mathrm{Ni}, \mathrm{Cu}$ and $\mathrm{Zn}$ and trivalent ions such as $\mathrm{Cr}$ and Fe can take the places of $\mathrm{Mg}^{2+}$ and $\mathrm{Al}^{3+}$ respectively to yield a large number of LDHs. ${ }^{7}$ The general formula of an LDH may be represented as [M(II) $)_{1-x} \mathrm{M}^{\prime}(\mathrm{III})_{x}$ $\left.(\mathrm{OH})_{2}\right]\left(\mathrm{A}^{n-}\right)_{x / n} \cdot \mathrm{yH}_{2} \mathrm{O}$. The molecular formula of a carbonate containing LDH with $x=0.25$ is $\mathrm{M}_{6} \mathrm{M}_{2}$ $(\mathrm{OH})_{16} \mathrm{CO}_{3} \cdot 4 \mathrm{H}_{2} \mathrm{O}$. In this paper we designate the LDHs as M-M'-A.

Most preparations of LDHs exhibit PXRD patterns with excessive and non-uniform broadening of lines which cannot readily be used for structure elucidation. As an illustration we study the $\mathrm{Mg}-\mathrm{Fe}-$ $\mathrm{CO}_{3}^{2-} \mathrm{LDH}$, the mineral form of which is known as pyroaurite. In this work, we examine the effect of different kinds of disorder on the PXRD pattern of the LDH by the use of DIFFaX simulations. DIFFaX is a fortran code that enables the simulation of the PXRD pattern of any given solid. ${ }^{8}$ Within the DIFFaX formalism ${ }^{9}$ a crystalline solid is treated as a stacking of sheets of atoms interconnected by stacking vectors - an approach that is ideally suited for layered materials. By using more than one stacking vector, different kinds of structural disorders can be engineered into the crystal and the resulting changes in the PXRD pattern can be simulated. Crystallite size effects can also be simulated for an ordered crystal. A comparison of the observed with the simulated patterns enables us to find possible causes for the non-uniform broadening of reflections in the observed patterns. Other authors have studied the nature of disorder in synthetic hydrotalcite and the $\mathrm{Mg}-\mathrm{Ga}$ $\mathrm{LDH}^{10}$ We also study the origin of non-uniform broadening of lines in the PXRD patterns of synthetic $\mathrm{SO}_{4}^{2-}$ containing $\mathrm{Mg}-\mathrm{Fe} \mathrm{LDHs}$ to examine the effect of anions in producing structural disorder.

\section{Experimental}

All preparations were carried out using a Metrohm Model 718 STAT titrino operating in the $\mathrm{pH}$ stat mode. The $\mathrm{Mg}-\mathrm{Fe}-\mathrm{CO}_{3}^{2-} \mathrm{LDH}$ corresponding to the composition $\left[\mathrm{Mg}_{0.75} \mathrm{Fe}_{0.25}(\mathrm{OH})_{2}\left(\mathrm{CO}_{3}\right)_{0.125}\right] \cdot \mathrm{yH}_{2} \mathrm{O}$ was prepared by adding a mixed metal $\left(\mathrm{Mg}^{2+}+\mathrm{Fe}^{3+}\right) \mathrm{ni}-$ trate solution $(50 \mathrm{ml})$ containing $0.051 \mathrm{~mol} \mathrm{\textrm {Mg } ^ { 2 + }}$ and $0.017 \mathrm{~mol} \mathrm{Fe}^{3+}$ to $100 \mathrm{ml} \mathrm{Na}_{2} \mathrm{CO}_{3}$ solution containing three times the stoichiometric requirement of $\mathrm{CO}_{3}^{2-}$ ions under stirring conditions. Simultaneously a $1.5 \mathrm{M} \mathrm{NaOH}$ solution was dispensed to maintain the $\mathrm{pH}$ at 10 . The slurry was aged at $90^{\circ} \mathrm{C}$ for $18 \mathrm{~h}$, washed free of $\mathrm{OH}^{-}$ions and dried at $65^{\circ} \mathrm{C}$.
$\mathrm{Mg}-\mathrm{Fe}-\mathrm{SO}_{4}^{2-} \mathrm{LDH}$ corresponding to the formula $\left[\mathrm{Mg}_{0.75} \mathrm{Fe}_{0.25}(\mathrm{OH})_{2}\right]\left(\mathrm{SO}_{4}\right)_{0.125} \cdot \mathrm{yH}_{2} \mathrm{O}$ was prepared by the procedure described by Khaldi et al. ${ }^{11}$ A mixed metal nitrate solution containing $\mathrm{Mg}^{2+}$ and $\mathrm{Fe}^{3+}$ in the required stoichiometric amount was added to a $\mathrm{Na}_{2} \mathrm{SO}_{4}$ solution at $65^{\circ} \mathrm{C}$ containing seven times the stoichiometric requirement of $\mathrm{SO}_{4}^{2-}$ ions. Simultaneously, $1 \mathrm{~N} \mathrm{NaOH}$ solution was dispensed to maintain the $\mathrm{pH}$ at 10 . All solutions were prepared using decarbonated water. The slurry was aged at $65^{\circ} \mathrm{C}$ for 3 days under conditions of constant stirring and purging with nitrogen gas. The precipitate was washed with copious amounts of hot water rinsed with acetone and dried at $65^{\circ} \mathrm{C}$ overnight.

PXRD patterns were recorded on a Philips X'pert powder diffractometer equipped with a filter to cut off fluorescence radiation $(\mathrm{Cu} \mathrm{K \alpha}$ source, $\lambda=1.541 \AA)$ at a continuous scan rate of $2^{\circ} 2 \theta \mathrm{min}^{-1}$ and the data was rebinned into steps of $0.05^{\circ}$. The instrumental broadening of the Bragg peaks is estimated to be $0 \cdot 15-0 \cdot 2^{\circ} 2 \theta$ in the range $77-28^{\circ} 2 \theta$ for the Si standard.

To confirm the presence of intercalated anions, IR spectra of all the samples were recorded (Nicolet model Impact 400D FTIR spectrometer, 4000$400 \mathrm{~cm}^{-1}$; resolution $4 \mathrm{~cm}^{-1}, \mathrm{KBr}$ pellet).

\section{DIFFaX simulations}

The details of the DIFFaX simulations of LDHs are described in detail elsewhere ${ }^{12}$ and briefly summarized here.

The LDH layer comprises a brucite-like hydroxide sheet and an interlayer of anions and water molecules. The composition of the hydroxide sheet is $\left[\mathrm{MO}_{2}\right]$ and that of the interlayer $\mathrm{C}_{x / 2} \mathrm{O}_{z}$ or $\mathrm{S}_{x / 2} \mathrm{O}_{z}$ depending on the anion ( $x=$ trivalent ion content). In the case of $\mathrm{CO}_{3}^{2-}$ anions, the oxygen atoms of the carbonate ions and water molecules occupy a single set of sites, ${ }^{13}$ so that, of the $z \mathrm{O}$ atoms, $3 x / 2$ belong to the carbonate ions and $(z-3 x / 2)$ correspond to the water content of the interlayer. Similarly the basal oxygen atoms of the $\mathrm{SO}_{4}^{2-}$ ion share a common set of sites with the $\mathrm{O}$ atoms of the interlayer water, while the apical oxygen occupies another set of sites, ${ }^{14}$ hence of the $z \mathrm{O}$ atoms, $4 x / 2$ belong to the sulphate ions and $(z-4 x / 2)$ correspond to the interlayer water content. By varying $z$, the water content in the LDH can be varied.

The $\mathrm{Mg}-\mathrm{Fe}-\mathrm{CO}_{3}^{2-} \mathrm{LDH}$ layer was defined using the single crystal data obtained from the Internatio- 
nal Crystal Structure Database (ICSD No. 6295). Since no ICSD data exist for the $\mathrm{Mg}-\mathrm{Fe}-\mathrm{SO}_{4}^{2-} \mathrm{LDH}$, the ICSD data of related LDHs were used to create the input files. For the $1 \mathrm{H}$ polytype, the ICSD No. $91860\left(\mathrm{Zn}-\mathrm{Al}-\mathrm{SO}_{4}^{2-}\right), 2 \mathrm{H}_{1}$ polytype ICSD No. 75542 $\left(\mathrm{Zn}-\mathrm{Cr}-\mathrm{SO}_{4}^{2-}\right)$ and the $3 \mathrm{R}_{1}$ polytype ICSD No. $91859\left(\mathrm{Zn}-\mathrm{Al}-\mathrm{SO}_{4}^{2-}\right)$ was used. Where no comparison with experimental patterns is required, the calculated Bragg reflections were broadened by using a Lorentzian line shape (FWHM $0 \cdot 2^{\circ} 2 \theta$ ) to simulate the effect of instrumental broadening. Where a comparison with an experimental pattern is intended, the calculated peaks were broadened using a Lorentzian having the same FWHM as that of the 110 reflection in the observed pattern. Broadening of the 110 reflection in excess of the instrumental factors is ascribed to crystallite size effects as this reflection is largely unaffected by structural disorder. ${ }^{12}$ The stacking vector $(0,0,1)$ generates the $1 H$ polytype while $(2 / 3,1 / 3,1 / 3)$ generates the $3 R_{1}$ polytype. The $2 H_{1}$ polytype is generated by defining two different layers $A C$ and $C A$ and then stacking them one atop another using the stacking vector $(0,0,1 / 2)$.

Disorder is built into the lattice by using more than one stacking vector with different probabilities. A stacking vector $\left(x, y, c_{o}\right)$ ( $x, y$ are random numbers) generates turbostratic disorder. $(0,0, r)\left(r=c / c_{o}\right)$ simulates the interstratification of a layer with thickness $c$ into a crystal with periodicity $c_{o}$. Stacking faults are incorporated in the crystal by the simultaneous use of vectors $\left(0,0, c_{o}\right)$ with $\left(1 / 3,2 / 3, c_{o}\right)$ and $(2 / 3$, $1 / 3, c_{o}$ ) with different probabilities. The probabilities of the stacking vectors are varied by hand until a good visual match between the simulated and experimental patterns is obtained. The goodness of the visual match is based on obtaining an approximate match of (1) the peak positions $\left( \pm 0 \cdot 1^{\circ}\right.$ in $\left.2 \theta\right)$, (2) FWHM values $\left( \pm 0 \cdot 1^{\circ}\right.$ in $\left.2 \theta\right)$ and (3) intensities $( \pm 5 \%)$ of the simulated peaks with those in the observed pattern. To facilitate this comparison, the experimental and simulated patterns are overlaid.

Crystallite size is defined in the $a-b$ plane as 'disc diameter' and along the $c$-crystallographic axis as 'thickness'.

\section{Results and discussion}

The observed PXRD patterns of the Mg-Fe-A ( $\mathrm{A}=$ $\left.\mathrm{CO}_{3}^{2-}, \mathrm{SO}_{4}^{2-}\right) \mathrm{LDHs}$ are given in figure 1 . In figure 2 are given the DIFFaX simulated patterns obtained from model single crystal structures reported for the carbonate and sulphate containing LDHs. The observed and expected peak positions together with the FWHM values are listed in table 1. The expected Bragg reflections chiefly belong to the following types:

(1) The basal reflections indexed as $00 \ell$. These reflections appear in the low $\left(<20^{\circ} 2 \theta\right)$ angle region and depend on the size of the intercalated anion.

(2) The $h 0 / / 0 k l$ family of reflections appear in the mid- $2 \theta\left(30-50^{\circ}\right)$ region. As is evident from figure 2, the different model LDH structures can be distinguished from each other by these reflections.

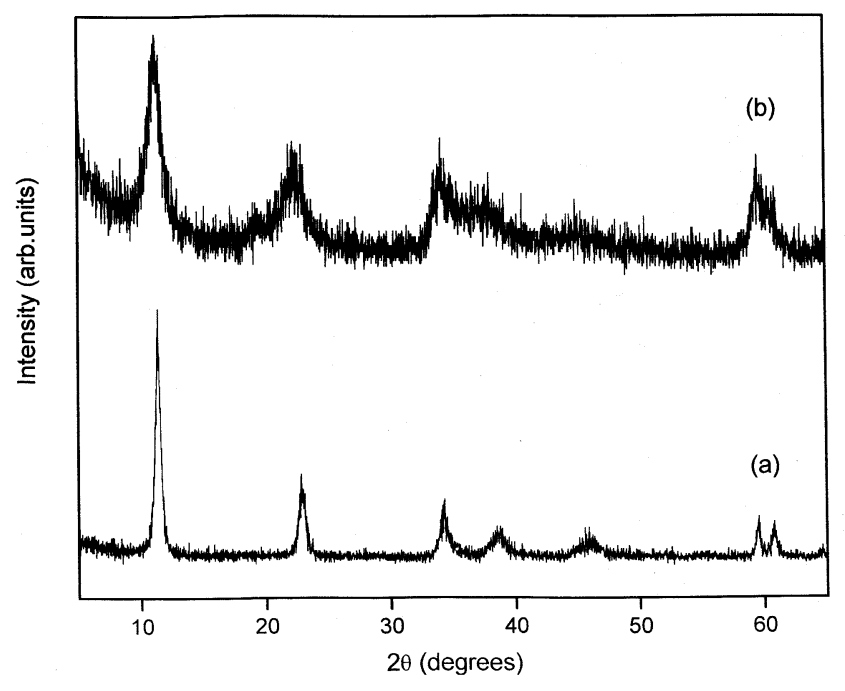

Figure 1. PXRD patterns of the as-prepared LDHs. (a) $\mathrm{Mg}-\mathrm{Fe}-\mathrm{CO}_{3}^{2-}$ (b) $\mathrm{Mg}-\mathrm{Fe}-\mathrm{SO}_{4}^{2-}$.

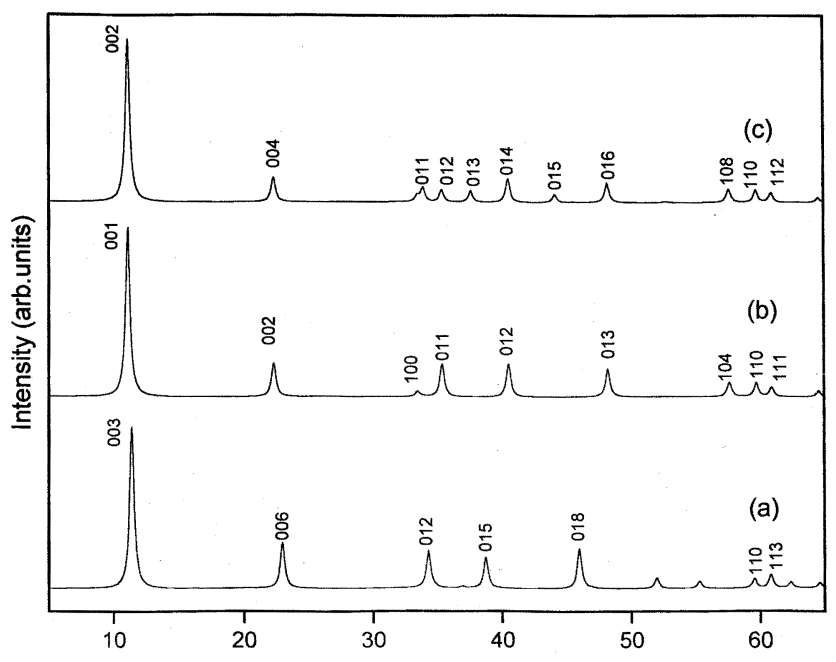

Figure 2. DIFFaX simulated PXRD patterns of model structures of (a) triple-layered $\mathrm{CO}_{3}^{2-}$ containing $\mathrm{LDH}$, (b) single-layered and (c) double-layered $\mathrm{SO}_{4}^{2-}$ containing LDH. A triple-layered $\mathrm{SO}_{4}^{2-}$ containing $\mathrm{LDH}$ has a similar pattern to (a). 
Table 1. A comparison of the Bragg angles expected for different model structures with the observed pattern.

\begin{tabular}{|c|c|c|c|c|c|c|}
\hline \multicolumn{3}{|c|}{$\mathrm{LDH}-\mathrm{CO}_{3}^{2-}$} & \multicolumn{4}{|c|}{ LDH-SO ${ }_{4}^{2-}$} \\
\hline$h k l$ & $3 R_{1}$ & Exp* & $h k l$ & $1 H$ & $2 H_{1}$ & Exp* \\
\hline 003 & 11.4 & $11.4(0.5)$ & 001 & $11 \cdot 1$ & & \\
\hline 006 & 22.95 & $22.9(0.6)$ & 002 & $22 \cdot 3$ & $11 \cdot 1$ & $11 \cdot 1(1 \cdot 4)$ \\
\hline 012 & $34 \cdot 2$ & $34.2(0.55)$ & 004 & & $22 \cdot 3$ & $22 \cdot 3(2 \cdot 1)$ \\
\hline 015 & 38.65 & $38.50(1.2)$ & 011 & $35 \cdot 3$ & 33.9 & $33.9(\mathrm{vb})$ \\
\hline \multirow[t]{4}{*}{018} & 45.95 & $45 \cdot 70(1 \cdot 3)$ & 012 & $40 \cdot 5$ & & \\
\hline & & & 013 & $48 \cdot 2$ & & \\
\hline & & & 014 & & $40 \cdot 5$ & \\
\hline & & & 016 & & $48 \cdot 2$ & \\
\hline 110 & $59 \cdot 5$ & $59.5(0.35)$ & 110 & $59 \cdot 7$ & 59.7 & $59.7(\mathrm{vb})$ \\
\hline \multirow[t]{2}{*}{113} & $60 \cdot 8$ & $60 \cdot 8(0 \cdot 35)$ & 111 & $60 \cdot 9$ & & \\
\hline & & & 112 & & $60 \cdot 9$ & \\
\hline
\end{tabular}

*Values in parantheses correspond to the FWHM in ${ }^{\circ} 2 \theta ;^{\dagger}$ very broad

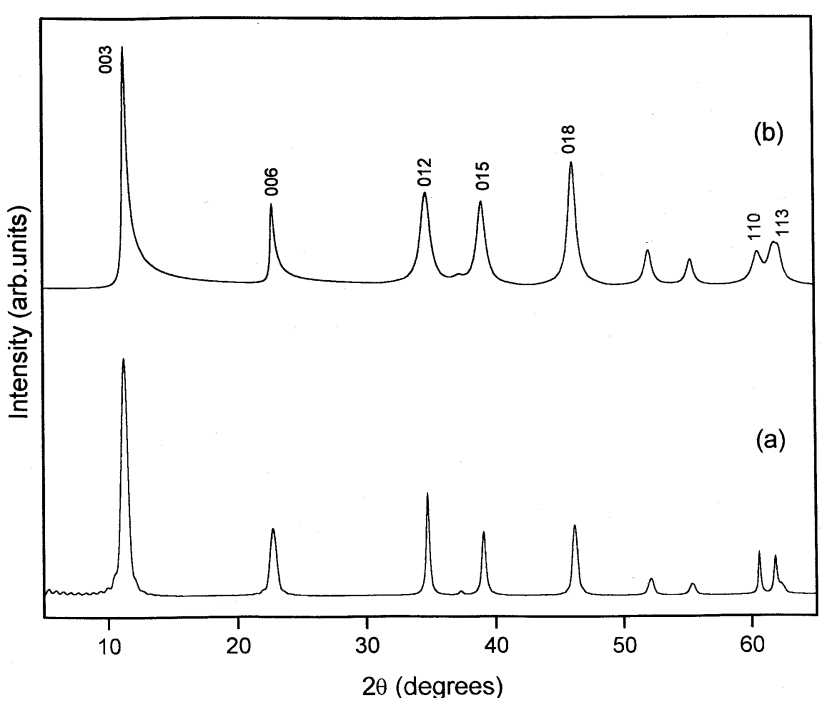

Figure 3. Crystallite size effects on the PXRD pattern of the $\mathrm{Mg}-\mathrm{Fe}-\mathrm{CO}_{3}^{2-} \mathrm{LDH}$. (a) Layer thickness, $15 \mathrm{~nm}(20$ layers). (b) Disc diameter, $30 \mathrm{~nm}$.

(3) The $h k 0$ and $h k l$ reflections appear in the high $\left(55-65^{\circ} 2 \theta\right)$ angle region. These reflections largely remain invariant in different LDHs.

It is evident that there are significant differences between the observed and simulated patterns. The sulphate-containing LDH in particular shows only four excessively broadened reflections. Such patterns have been reported by numerous authors (see refs. $[15,16]$ as representative illustrations). To account for these differences, we examine below the effect of different phenomena that can in principle lead to the broadening of PXRD patterns of LDHs. The dis- cussion is chiefly carried out with respect to the carbonate containing LDH.

\subsection{Crystallite size effects}

In layered solids, the intralayer bonding is typically iono-covalent in nature while the interlayer bonding is weaker. Crystal growth takes place preferentially in the $a-b$ plane resulting in anisotropic particles. Within the DIFFaX formalism, anisotropic particles are described by specifying a 'disc diameter' and 'thickness'. In figure 3 is shown the effect of particle size on the PXRD pattern of the $\mathrm{Mg}-\mathrm{Fe}-\mathrm{CO}_{3}^{2-} \mathrm{LDH}$. Restricting the disc diameter affects the 110 reflection the most. At $150 \mathrm{~nm}$ the 110 reflection is broadened to a FWHM value of $0 \cdot 35^{\circ} 2 \theta$, which is the experimentally observed value. At lower disc diameters, all the reflections are broadened, the least affected being the 00l. The latter however acquire an asymmetry, a feature not observed experimentally. As expected, a small thickness affects $00 \ell$ and $0 \mathrm{kl}$ families of reflections. Among these, the 012 reflection is broadened the least given its low $\ell$-weightage. In figure 4 are plotted the FWHM values of different reflections as a function of the crystallite thickness. The 110 reflection is unaffected. Restricting the thickness to $15 \mathrm{~nm}$ (20 layers) causes a broadening of the 003 reflection to a FWHM value of $0 \cdot 6^{\circ} 2 \theta$. When the thickness is reduced below this value additional low angle reflections (seen as weak oscillations in figure 3) are generated, probably due to "end to end' diffraction from the crystallites. Since such low angle reflections are not observed, the effect of 
particle size is confined to a broadening of the basal reflections up to a value of $0.6^{\circ} 2 \theta$ and the $0 \mathrm{kl}$ reflections up to $0 \cdot 3-0 \cdot 5^{\circ} 2 \theta$, corresponding to a particle thickness of $15 \mathrm{~nm}$. Any broadening of these reflections beyond this value is for other reasons.

\subsection{Interstratification}

The solubility product of $\mathrm{Mg}(\mathrm{OH})_{2}\left(5.5 \times 10^{-12}\right)$ is much higher than that of ' $\mathrm{Fe}(\mathrm{OH})_{3}$ ' $\left(3.8 \times 10^{-38}\right) .^{17}$ Consequently, there is always the possibility of the serial precipitation of the two unitary hydroxides during the preparation of the LDH. The unitary hy-

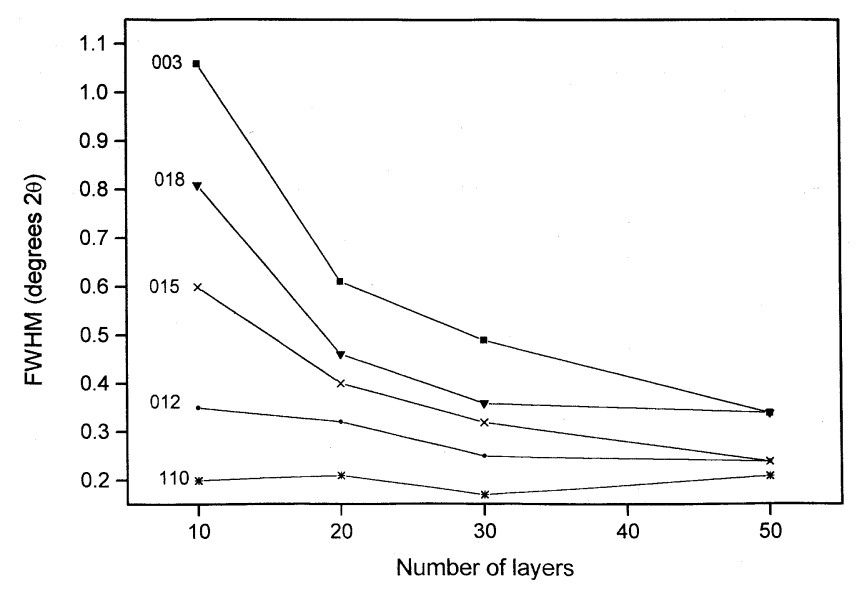

Figure 4. A plot of the FWHM as a function of layer thickness for the different Bragg reflections.

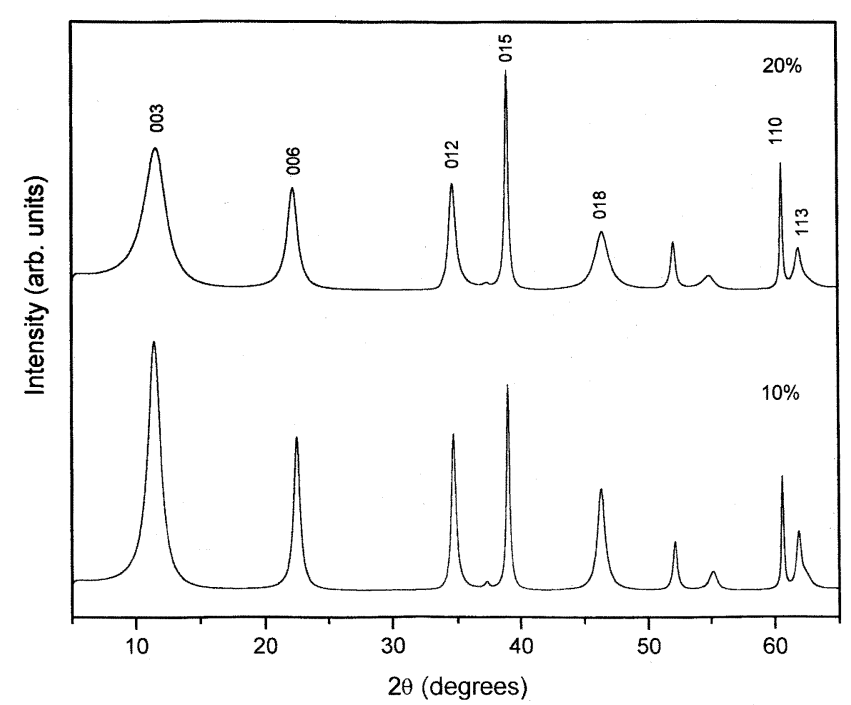

Figure 5. Simulated $\mathrm{PXRD}$ patterns of the $\mathrm{Mg}-\mathrm{Fe}-\mathrm{CO}_{3}^{2-}$ LDH interstratified with different percentages of $\mathrm{Mg}(\mathrm{OH})_{2}$. droxides have an interlayer distance of $4.7 \AA$. Interstratification of the unitary hydroxide within the matrix of the LDH leads to a non-integral repeat distance along the stacking direction. In figure 5, we show the effects of incorporating progressively increasing amounts of interstratification. Even a small proportion of interstratification broadens the basal reflections. A $20 \%$ interstratification broadens the 003 reflection to an FWHM value of $2^{\circ} 2 \theta$. Understandably the 110 reflection is unaffected. Surprisingly the 015 reflection is affected the least among the non- $h k 0$ reflections.

\subsection{Stacking disorder}

A consequence of the layered nature of the LDHs is that the metal hydroxide sheets can be stacked in various ways to obtain a large number of polytypes. Naturally occurring LDHs are known to crystallize in either a triple layer cell with rhombohedral symmetry designated as $3 R$ (see figure $2 \mathrm{a}$ for the corresponding PXRD pattern) or a double layer cell with hexagonal symmetry designated as $2 \mathrm{H}$ (figure 2c). ${ }^{18,19}$ For example $\mathrm{Mg}_{6} \mathrm{Fe}_{2}(\mathrm{OH})_{16} \mathrm{CO}_{3} \cdot 4 \mathrm{H}_{2} \mathrm{O}$ exists in its mineral form as pyroaurite $\left(3 R_{1}\right)$ or sjogrenite $\left(2 H_{1}\right)$ respectively. ${ }^{13}$ Bookin and coworkers ${ }^{18,19}$ have derived the complete list of polytypes among the LDHs by both experiment and theory. Using symbols $\mathrm{A}, \mathrm{B}$ and $\mathrm{C}$ to represent hydroxyl ion positions and symbols $\mathrm{a}, \mathrm{b}$ and $\mathrm{c}$ to represent cation positions, a typical metal hydroxide sheet can be represented as $\mathrm{AbC}$ or $\mathrm{AC}$. The stacking sequences of the most common LDH polytypes are listed below.

\section{$1 H \quad \mathrm{AC} \mathrm{AC}-----$ \\ $3 R_{1} \quad \mathrm{AC}$ CB BA AC----- \\ $2 H_{1} \quad$ AC CA AC-----}

The carbonate ions occupy the interlayer sites. In the $1 H$ polytype the interlayer sites are octahedral, while in the other two the interlayer sites are prismatic. Carbonates are known to prefer prismatic sites ${ }^{13}$ and this explains the preponderance of the $3 R_{1}$ and $2 H_{1}$ polytypes among naturally occurring and synthetic LDHs. There are some instances of sulphate-containing LDHs crystallizing in the $1 H$ polytype (figure $2 \mathrm{~b}) .^{19}$

Interlayer distance depends upon the van der Waal's radius of the intercalated anion and remains 
unchanged in different polytypes containing the same anion. The metal hydroxide sheet also remains unchanged in different LDH systems. Consequently, the $00 \ell$ and the $h k 0$ reflections remain invariant in different polytypes. The different polytypes can be distinguished from one another by the positions and intensities of the $h 0 / / 0 \mathrm{kl}$ family of Bragg reflections appearing in the mid- $2 \theta\left(30-55^{\circ}, \mathrm{Cu} \mathrm{K} \alpha\right)$ region of the PXRD pattern. ${ }^{18,19}$ When stacking disorders arise due to the intergrowth of two or more polytypes, the $h 0 l / 0 \mathrm{kl}$ reflections are affected the most. As an illustration in figure 6 is shown the effect of incorporating

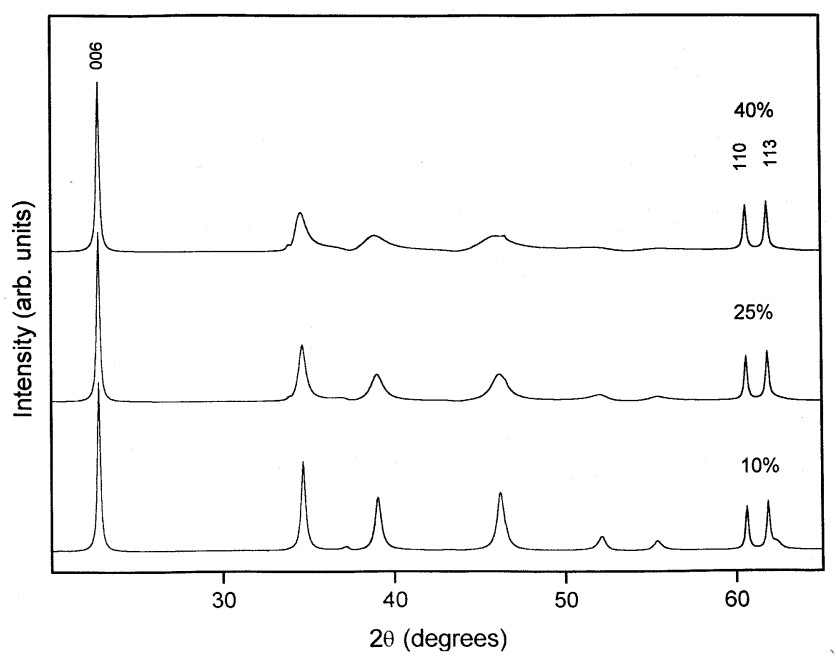

Figure 6. Simulated PXRD patterns of the $3 \mathrm{R}_{1}$ polytype of the $\mathrm{Mg}-\mathrm{Fe}-\mathrm{CO}_{3}^{2-} \mathrm{LDH}$ with different proportions of the $2 \mathrm{H}_{1}$ motif. For reasons of clarity, the low angle region is not shown.

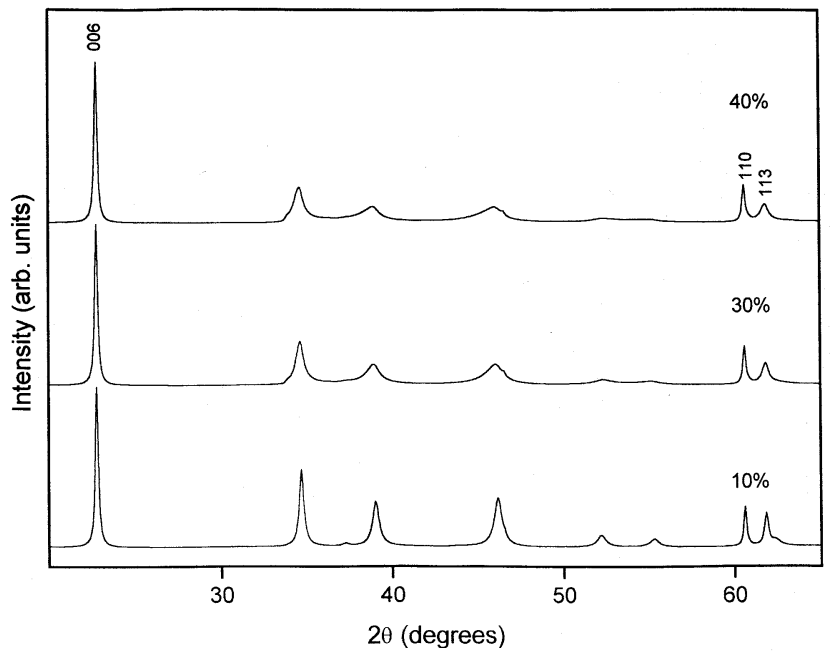

Figure 7. Simulated PXRD patterns of the $\mathrm{Mg}-\mathrm{Fe}-\mathrm{CO}_{3}^{2-}$ $\mathrm{LDH}$ with varying proportions of turbostraticity. For reasons of clarity the low angle region is not shown. different proportions of $2 H_{1}$ stacking motifs within the $3 R_{1}$ polytype.

\subsection{Turbostraticity}

Turbostratic disorder arises out of the random orientation of successive layers about the stacking direction, leading to a loss in registry between successive hydroxide layers. Introduction of even small percentages of turbostratic disorder $(10 \%)$ drastically broadens the $0 k l$ reflections while it does not affect the $00 \ell$ and 110 reflections (figure 7). While these effects are some what similar to those arising out of stacking faults, turbostraticity additionally affects the 113 reflection.

\subsection{Simulation of the experimental patterns}

With this understanding we then examined the observed PXRD patterns of the $\mathrm{Mg}-\mathrm{Fe}$ LDHs. The PXRD pattern of the carbonate containing LDH could be simulated by incorporating stacking disorders. Inclusion of $20 \% 2 H_{1}$ motifs in the $3 R_{1}$ matrix yielded a good match with the experimental pattern. The PXRD pattern of the sulphate containing LDH was generated by the inclusion of $40 \%$ turbostratic disorder within the $2 H_{1}$ matrix. A comparison of the experimental and simulated patterns is shown in figure 8. No attempt was made to fit the relative intensity of the 003 reflection in the sulphate containing $\mathrm{LDH}$ as this is known to be sensitive to the interlayer

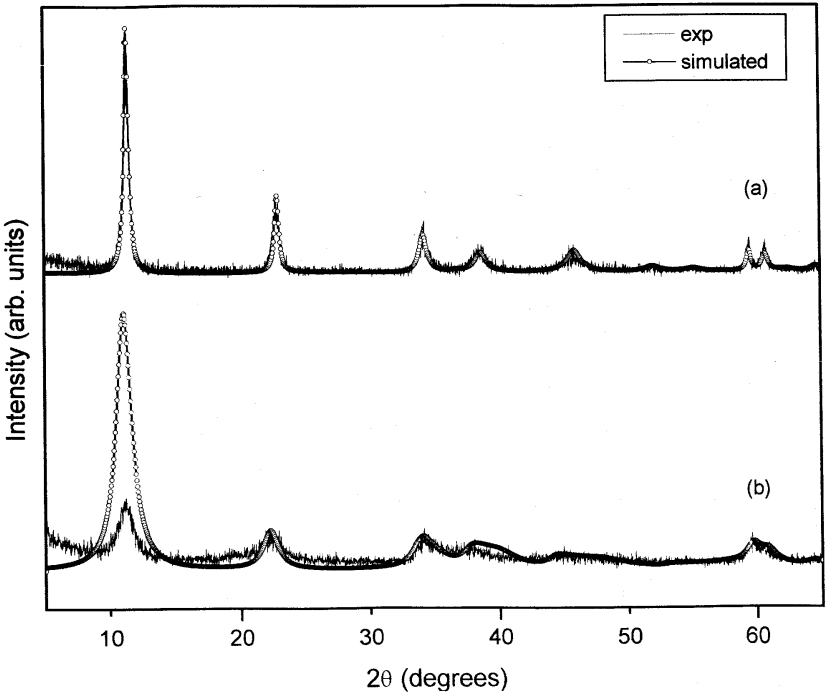

Figure 8. Results of the simulations of the experimental patterns of (a) the as-prepared $\mathrm{Mg}-\mathrm{Fe}-\mathrm{CO}_{3}^{2-}$ and (b) the as-prepared $\mathrm{Mg}-\mathrm{Fe}-\mathrm{SO}_{4}^{2-} \mathrm{LDH}$. 
content. ${ }^{12}$ Even a small increase in the intercalated water content can change the relative intensities of the basal reflections.

\section{Conclusions}

In conclusion, while particle size effects contribute to the broadening of the Bragg reflections in LDHs, the type of excessive and non-uniform broadening seen in the observed patterns is most likely on account of structural disorder. DIFFaX simulations enable the classification and quantification of structural disorders. As in any simulation technique, there could be multiple solutions and hence the simulations reported here are illustrative rather than being definitively quantitative in character. Nevertheless, the pattern of non-uniform broadening is diagnostic of specific types of structural disorder. There are evidences that the physical and chemical properties of layered hydroxides are correlated to the nature and extent of structural disorder. ${ }^{20-22}$ There are significant challenges in synthesizing materials with specific kinds of disorder for different applications. The need for engineering disorder into materials for specific applications was first pointed out by Ovshinsky, Fetchenko and Ross ${ }^{23}$ albeit in a different class of materials. Correlation of structural disorder with materials properties would be of interest in many more classes of materials.

\section{Acknowledgement}

The authors thank the Department of Science and Technology, Govt. of India for financial support. GST thanks the University Grants Commission, New Delhi for support under the Faculty Improvement Programme. The authors thank the Solid State and Structural Chemistry Unit, Indian Institute of Science for powder X-ray diffraction facilities. PVK thanks Prof R Seshadri for introducing him to the DIFFaX technique and Prof T N Guru Row for useful discussions.

\section{References}

1. West A R 1998 Solid state chemistry and its applications (New Delhi: John Wiley and Sons) p. 173

2. Bernard M C, Cortes R, Keddam M, Takenouti H, Bernard P and Senyarich S 1996 J. Power Sources 63 247

3. Ding Y, Zhang G, Wu H, Hai B, Wang L and Qian Y 2001 Chem. Mater. 13435

4. Radha A V, Kamath P V and Subbanna G N 2003 Mater. Res. Bull. 38731

5. Ramesh T N, Jayashree R S and Kamath P V 2003 Clays Clay Miner. 51570

6. Cavani F, Trifiro F and Vaccari A 1991 Catal. Today 11173

7. Carrado K A, Kostapapas A and Suib S L 1988 Solid State Ionics $\mathbf{2 6} 77$

8. Treacy M M J, Deem M W and Newsam J M Computer Code DIFFaX, Version 1.807

9. Treacy M M J, Newsam J M and Deem M W 1991 Proc. R. Soc. London A433 499

10. Bellotto M, Rebours B, Clause O, Lynch J, Bazin D and Elkaim E 1996 J. Phys. Chem. 1008527

11. Khaldi M, de Roy A, Chaouch M and Besse J P 1997 J. Solid State Chem. 13066

12. Thomas G S, Rajamathi M and Kamath P V 2004 Clays Clay Miner. 52693

13. Taylor H F W 1973 Miner. Mag. 39377

14. Ennadi A, Khaldi M, de Roy A and Besse J P 1994 Mol. Cryst. Liq. Cryst. 244373

15. Zhao Y, Li F, Zhang R, Evans D G and Duan X 2002 Chem. Mater. 144286

16. Kloprogge J T, Wharton D, Hickey L and Frost R L 2002 Am. Miner. 87623

17. Dobos D 1975 Electrochemical data. A handbook for electrochemists in industry and universities (Amsterdam: Elseivier Scientific) p. 221

18. Bookin A S and Drits V A 1993 Clays Clay Miner. 41551

19. Bookin A S, Cherkashin V I and Drits V A 1993 Clays Clay Miner. 41558

20. Ramesh T N, Kamath P V and Shivkumara C 2005 J. Electrochem. Soc. 152 A806

21. Jayashree R S and Kamath P V 2002 J. Electrochem. Soc. 149 A761

22. Jayashree R S, Kamath P V and Subbanna G N 2000 J. Electrochem. Soc. 1472029

23. Ovshinsky S R, Fetcenko M A and Ross J 1993 Science 260176 